\title{
Electronic Structure, Optical and Magneto-Optical Properties and X-Ray Magnetic Circular Dichroism in Hybrid Heterostructures $\mathrm{Sr}_{2} \mathrm{CrReO}_{6} / \mathrm{BaTiO}_{3}$
}

\author{
V.N. Antonov ${ }^{a, b}$, L.V. BeKenov ${ }^{a}$, S. $\mathrm{UBA}^{b, *}$, A. BondA ${ }^{b}$ And L. UBA ${ }^{b}$ \\ ${ }^{a}$ G.V. Kurdyumov Institute for Metal Physics of the N.A.S. of Ukraine, 36 Vernadsky Street, 03142 Kiev, Ukraine \\ ${ }^{b}$ Faculty of Mathematics and Informatics, University of Bialystok, K. Ciolkowskiego 1M, PL-15245 Bialystok, Poland

\begin{abstract}
We report on the electronic structure of hybrid heterostructure combined the ferromagnetic double perovskite $\mathrm{Sr}_{2} \mathrm{CrReO}_{6}$ (SCRO) and ferroelectric $\mathrm{BaTiO}_{3}$ (BTO) calculated in the GGA approach using the fully relativistic spin-polarized Dirac LMTO method. The optical and MO properties as well as the X-ray absorption spectra and Xray magnetic circular dichroism at the $\mathrm{Ti}, \mathrm{Ba}$, and $\mathrm{Re} L_{2,3}$ edges and $\mathrm{O} K$ edges of the $\mathrm{SCRO} / \mathrm{BTO}$ heterostructures were investigated theoretically from the first principles. It was shown that the calculations reproduce well available experimental spectra and allow to explain the microscopic origin of the SCRO/BTO heterostructures optical, MO and XMCD spectra.
\end{abstract}

DOI: 10.12693/APhysPolA.133.456

PACS/topics: 75.50.Cc, 71.20.Lp, 71.15.Rf

\section{Introduction}

The phenomena ferroelectricity and ferromagnetism derive from the fact that the electrons possess charge and spin. Depending on the surroundings, a material may have an electric polarization or a magnetic polarizations. A few materials possess both ferroelectric and magnetic polarizations in the same phase and a coupling between them. These materials are called multiferroics and possess so called magneto-electric (ME) effect, meaning magnetic (electric) induction of polarization $P$ (magnetization $M$ ) [1]. Multiferroics offer rich physics and novel devices concepts, however, the number of multiferroics offering a perspective for applications is rare. In fact, $\mathrm{BiFeO}_{3}$ is the only room temperature multiferroic (antiferromagnetic and ferroelectric) so far, which has attracted great interest and extensive studies in the past decade [2]. Recently, much effort has been made to synthesis heterostructure thin films composed of materials that have large ferromagnetic (FM) and ferroelectric (FE) ordering, with the possibility of ME coupling.

Most technologically important ferroelectrics such as $\mathrm{BaTiO}_{3}(\mathrm{BTO})$ and $(\mathrm{Pb}, \mathrm{Zr}) \mathrm{TiO}_{3}$ are transition metal oxides. In parallel, there is a large number of ferromagnetically ordered ferrites with a spinel structure $\left(\mathrm{AB}_{2} \mathrm{O}_{4}\right)[3]$ and double perovskites $\mathrm{A}_{2} \mathrm{BB}^{\prime} \mathrm{O}_{6}(\mathrm{~A}=$ alkaline earth or rare earth and $\mathrm{BB}^{\prime}$ are transition metals such as $\mathrm{B}=\mathrm{Fe}$, $\left.\mathrm{Cr}, \mathrm{Mn}, \mathrm{Co}, \mathrm{Ni} ; \mathrm{B}^{\prime}=\mathrm{Mo}, \mathrm{Re}, \mathrm{W}\right)$ [4]. Recently, Opel et al. [5] have grown heteroepitaxial hybrid structures of $\mathrm{Sr}_{2} \mathrm{CrReO}_{6}$ (SCRO) on BTO by laser-MBE. A strong coupling between the ferroelectric order parameter in $\mathrm{BTO}$ and the ferromagnetic order parameter in SCRO

*corresponding author; e-mail: uba@uwb.edu.pl has been shown. That is, the SCRO/BTO heterostructure can be considered as a magneto-electric multiferroic.

The aim of this work is the detailed theoretical investigations of the electronic structure, optical, magnetooptical (MO) properties and X-ray magnetic circular dichroism (XMCD) in SCRO/BTO heterostructures.

\section{Computational details}

The details of the computational method are described in our previous papers [6-10], and here we only mention some aspects specific to the present calculations. The calculations presented in this work were performed using the spin-polarized fully relativistic linear-muffin-tin-orbital (SPR-LMTO) method [11]. The exchange-correlation functional of a GGA-type was used [12]. The X-ray absorption spectra (XAS) and X-ray magnetic dichroism (XMCD) were calculated taking into account the exchange splitting of core levels. Figure 1 shows the heterostructures used in our calculations.

\section{Results and discussion}

The GGA approach produces the half-metallic states in all the structures under consideration where the only contribution around the Fermi level comes from the minority spins. There is an energy gap of $0.45 \mathrm{eV}$, $0.80 \mathrm{eV}$, and $0.70 \mathrm{eV}$, between the $\mathrm{Cr} e_{g}$ and $\operatorname{Re} t_{2 g}$ states in the spin-up channel for SCRO, SCRO/BTO, and $2 \times[\mathrm{SCRO} / \mathrm{BTO}]$ heterostructures, respectively.

Figure 2 shows the experimentally measured [13] diagonal optical reflectivity spectra and dielectric functions of BTO (open magenta circles) and calculated spectra for $\mathrm{BTO}, \mathrm{SCRO}, \mathrm{SCRO} / \mathrm{BTO}$ heterostrucures.

The calculated optical reflectivity spectrum of SCRO has two deep local minima at $0.8 \mathrm{eV}$ and $2.8 \mathrm{eV}$. The 


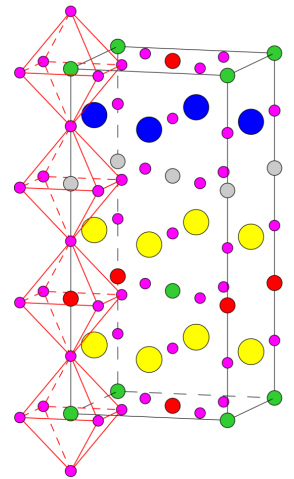

(c)

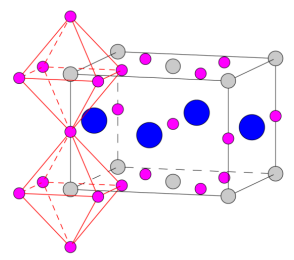

(b)

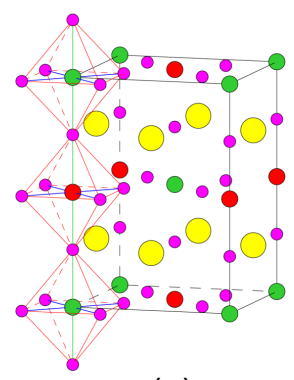

(a)

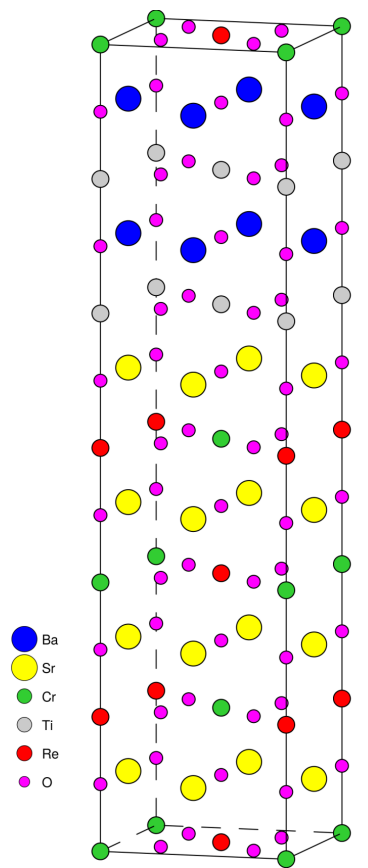

(e)

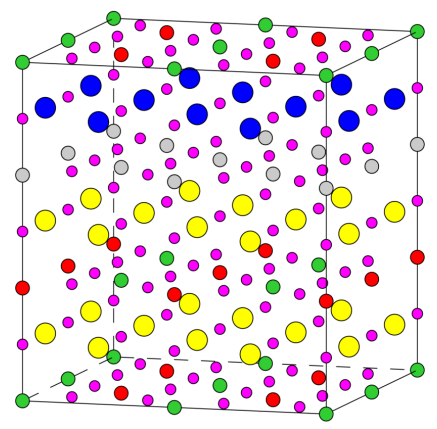

(d)

Fig. 1. schematic representation of SCRO structure (a), BTO structure (b), and SCRO/BTO $(1 \times 1 \times 1)(\mathrm{c})$, SCRO/BTO $(2 \times 2 \times 1)(\mathrm{d})$, and $\mathrm{SCRO} /(\mathrm{BTO}(1 \times 1 \times$

2) (e) heterostructures.

absorptive part of the dielectric function, $\varepsilon_{2 x x}(\omega)$, shows strong peak at around $1 \mathrm{eV}$. The optical reflectivity spectrum for the SCRO/BTO heterostructure has deeper minimum at $0.8 \mathrm{eV}$ in comparison with SCRO spectrum, besides, the minimum at $2.3 \mathrm{eV}$ has red shift by approximately $0.5 \mathrm{eV}$. The strong peak appeared at $1 \mathrm{eV}$ in the $\varepsilon_{2 x x}(\omega)$ dielectric function of SCRO perovskite is significantly reduced in the $\mathrm{SCRO} / \mathrm{BTO}$ heterostructure.

The Kerr rotation spectrum for SCRO possesses a strong peak about $2^{\circ}$ at the $3.5 \mathrm{eV}$ (not shown). There is no such strong peak in the Kerr rotation in SCRO/BTO heterostructures at the same energy. On the other hand, Kerr rotation spectra in $\mathrm{SCRO} / \mathrm{BTO}$ heterostructures have strong resonance peaks in infrared energy interval $0.3 \div 0.7 \mathrm{eV}$ mostly due to the plasma edge effect.

Figure 3 presents experimental XAS of BTO at the Ti $L_{2,3}$ edges [14] together with the spectra calculated in the GGA approximation in BTO and SCRO/BTO het-

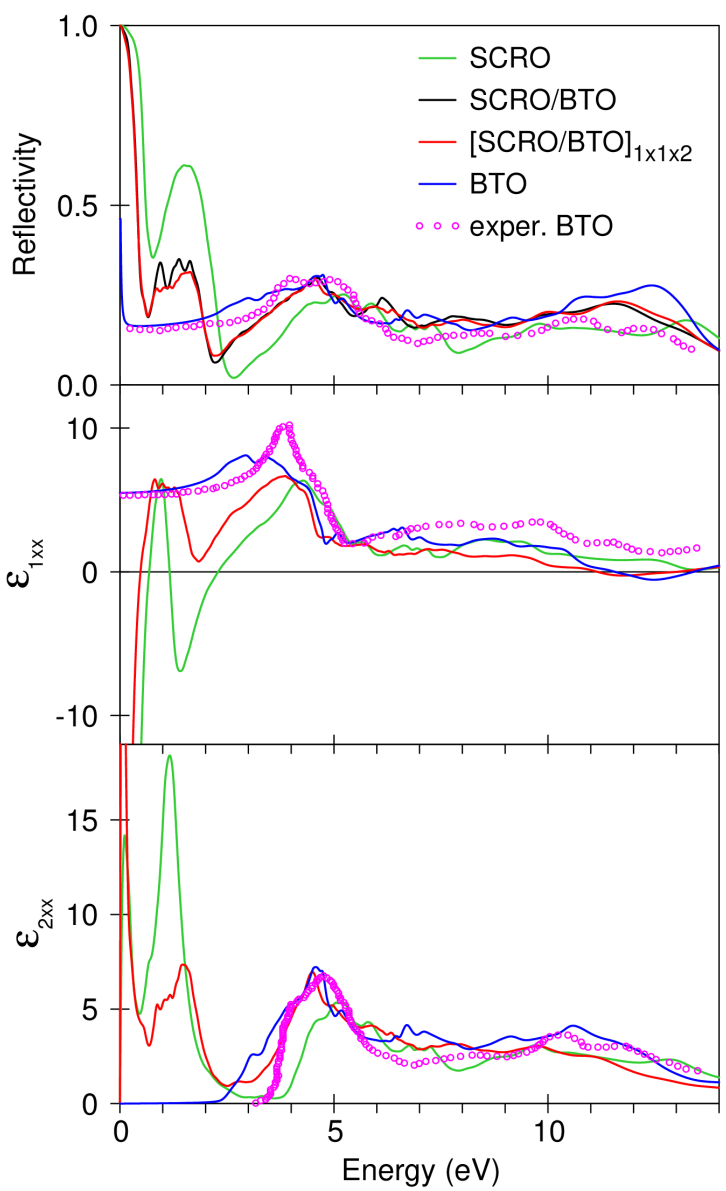

Fig. 2. The experimentally measured [13] diagonal optical reflectivily spectra (upper panel) and dielectric functions $\varepsilon_{1 x x}$ (middle panel), and $\varepsilon_{2 x x}$ (lower panel) of BTO (open magenta circles) and the spectra calculated by the SPR LMTO method for BTO (blue curves), SCRO double perovskite (green curves), SCRO/BTO monolayered $(1 \times 1 \times 1)$ heterostructure (black curve) and $(1 \times 1 \times 2)$ supercell heterostructure (red curves).

erostructure. The four experimentally observed intense peaks from 455 to $466 \mathrm{eV}$ can, to a first approximation, be assigned to $2 p_{3 / 2} \rightarrow e_{g}, 2 p_{3 / 2} \rightarrow t_{2 g}, 2 p_{1 / 2} \rightarrow e_{g}$, and $2 p_{1 / 2} \rightarrow t_{2 g}$ transitions, respectively. We found that the core-hole interactions significantly improve the agreement between theoretically calculated and experimentally measured Ti $L_{2,3}$ XAS spectra in BTO.

As expected for the $\mathrm{Ti}^{4+}$ ions and their octahedral coordination, BTO and SCRO/BTO heterostructure have similar spectra, as shown in Fig. 3. This demonstrates that there is no difference in the chemical environments of $\mathrm{Ti}$ ions. For the whole multilayered films, the $\mathrm{Ti}^{4+}$ is in octahedral coordination with oxygen, with all octahedra sharing corners. The XMCD spectra at the Ti $L_{3}$ and $L_{2}$ edges are well pronounced and show quite complicated shapes with several negative and positive peaks. The experimental measurements of the XMCD spectra at Ti $L_{2,3}$ edges in SCRO/BTO are highly desirable. 


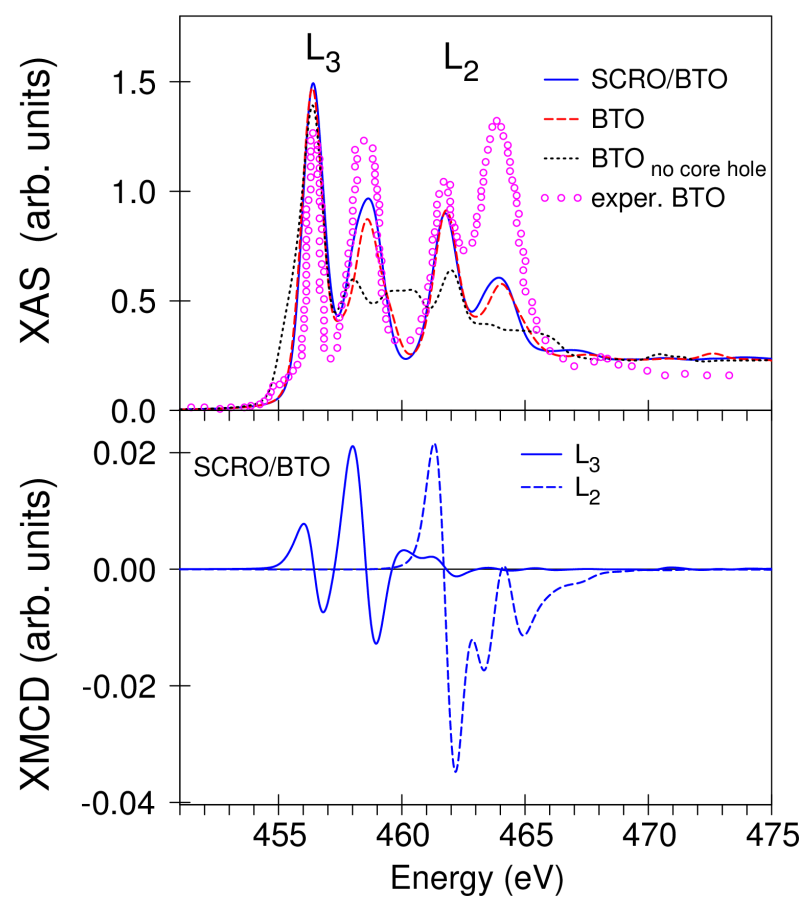

Fig. 3. Upper panel: the experimental X-ray absorption spectrum [14] (open circles) at the $\mathrm{Ti} L_{2,3}$ edges in BTO compared with the theoretically calculated ones for BTO with taking into account of core-hole effect (dashed red curve), BTO without taking into account of core-hole effect (dotted black curve), and SCRO/BTO heterostructure (full blue curve). Lower panel: theoretically calculated XMCD spectrum at the Ti $L_{2,3}$ edges in $\mathrm{SCRO} / \mathrm{BTO}$ heterostructure.

\section{Conclusions}

A systematic electronic structure study of SCRO/BTO heterostructures has been performed by employing the GGA approximation using the fully relativistic spinpolarized Dirac LMTO method. The electronic structure, optical and MO properties are studied in the BTO and SCRO oxides as well as in SCRO/BTO heterostructures. We found that without spin-orbit interactions SCRO and SCRO/BTO heterostructures possess half metallic state. The optical reflectivity spectrum for the SCRO/BTO heterostructure has deeper minimum at $0.8 \mathrm{eV}$ in comparison with SCRO spectrum, besides, the minimum at $2.3 \mathrm{eV}$ has red shift by approximately $0.5 \mathrm{eV}$. The Kerr rotation spectrum for SCRO shows a strong peak about $2^{\circ}$ at the $3.5 \mathrm{eV}$. There is no such strong peak in the Kerr rotation in SCRO/BTO heterostructures at this energy. However, Kerr rotation spectra in SCRO/BTO heterostructures have strong resonance peaks in infrared energy interval $0.3 \div 0.7 \mathrm{eV}$ due to the plasma edge effect. The theory reasonably well describes the X-ray absorption spectra in the BTO at the Ti $L_{2,3}$ edges. The final-state interaction improves the agreement between the theory and the experiment. We found that there is no difference in the chemical environments of Ti ions for the structures studied. For the whole multi- layered films, the $\mathrm{Ti}^{4+}$ is in octahedral coordination with oxygen, with all octahedra sharing corners.

Artificial oxide heterostructures are particularly promising for the realization of materials with improved and new functionalities and novel device concepts. Due to the complexity of the involved oxide materials, the rich variety of physics is far from being understood and needs further detailed studies. Moreover, intermixing of the different atomic species deposited in multilayer structures plays an important role and might influence or even dominate the overall physical properties. Therefore, a careful investigation of the electronic structure and physical properties of artificially constructed material systems is highly necessary.

\section{References}

[1] I.E. Dzyaloshinskii, Sov. Phys.-JETP 10, 628 (1959).

[2] J.T. Heron, D.G. Schlom, R. Ramesh, Appl. Phys. Rev. 1, 021303 (2014).

[3] P.R. Maiti, D. Chakravorty, Trans. Ind. Ceram. Soc. 70, 53 (2011).

[4] H. Kato, T. Okuda, Y. Okimoto, Y. Tomioka, K. Oikawa, T. Kamiyama, Y. Tokura, Phys. Rev. B 69, 184412 (2004).

[5] M. Opel, S. Gergäs, E.P. Menzel, A. Nielsen, D. Reisinger, K.-W. Nielsen, A. Brandlmaier, F.D. Czeschka, M. Althammer, M. Weiler, S.T.B. Goennenwein, J. Simon, M. Svete, W. Yu, S.-M. Hühne, W. Mader, R. Gross, Phys. Status Solidi A 208, 232 (2011).

[6] V.N.Antonov, M. Galli, F. Marabelli, A.N. Yaresko, A.Y. Perlov, E. Bauer, Phys. Rev. B 62, 1742 (2000).

[7] V.N.Antonov, B.N. Harmon, A.N. Yaresko, Phys. Rev. B 63, 205112 (2001).

[8] V.N. Antonov, L. Uba, S. Uba, A.N. Yaresko, A.Y. Perlov, V.V. Nemoshkalenko, Low Temp. Phys. 27, 425 (2001).

[9] V.N.Antonov, O. Jepsen, A.N. Yaresko, A.P. Shpak, J. Appl. Phys. 100, 043711 (2006).

[10] L. Uba, S. Uba, L.P. Germash, L.V. Bekenov, V.N. Antonov, Phys. Rev. B 85, 125124 (2012).

[11] A.Y. Perlov, A.N. Yaresko, V.N. Antonov, PYLMTO, A Spin-polarized Relativistic Linear Muffin-tin Orbitals Package for Electronic Structure Calculations (unpublished).

[12] J.P. Perdew, K. Burke, M. Ernzerhof, Phys. Rev. Lett. 78, 1396 (1997).

[13] G. Gupta, T. Nautiyal, S. Auluck, Phys. Rev. B 69, 052101 (2004).

[14] J. Zhang, A. Visinoiu, F. Heyroth, F. Syrowatka, M. Alexe, D. Hesse, H.S. Leipner, Phys. Rev. B 71, 064108 (2005). 